\title{
Proyecto de Innovación Docente: La Evaluación \\ Formativa y Compartida en Educación. Resultados de Transferencia de Conocimiento entre Universidad y \\ Escuela
}

\section{Teaching Innovation Project: Formative and Shared Assessment in Education. Results of Knowledge Transfer between University and School}

Cristina Pascual-Arias *

Víctor M. López-Pastor

Carolina Hamodi Galán

Universidad de Valladolid, España

\begin{abstract}
En este trabajo presentamos el desarrollo del Proyecto de Innovación Docente (PID) "La evaluación Formativa y compartida en educación. Transferencia de conocimiento entre Universidad y Escuela”, desarrollado en la Universidad de Valladolid. Hemos desarrollado un estudio de caso analizando la doble vía de transferencia de conocimiento: de la universidad a la escuela y de la escuela a la universidad. Para ello se han organizado dos líneas de intervención: (a) desarrollo de Proyectos de Aprendizaje Tutorados, periodos de Prácticum, Trabajos Fin de Grado, Trabajos Fin de Máster y Tesis Doctorales; (b) desarrollo un seminario internivelar sobre Evaluación Formativa y Compartida, como actividad de formación permanente para el profesorado de todas las etapas educativas, desde educación infantil hasta la universidad. Hemos obtenido como resultados ventajas, inconvenientes y propuestas de mejora para cursos posteriores desde el punto de vista del aprendizaje del alumno, la docencia del maestro y el propio proceso de enseñanza y aprendizaje.
\end{abstract}

Palabras clave: Evaluación formativa; Evaluación compartida; Transferencia de conocimiento; Formación permanente del profesorado; Formación inicial del profesorado.

In this work we present the development of the Teaching Innovation Project (TIP) "The Formative and shared assessment in education. Transfer of knowledge between University and School ", developed at the University of Valladolid, specifically in the Faculty of Education of Segovia. We have developed a case study analyzing the double way of knowledge transfer: from the university to the school and from the school to the university. To this end, two lines of intervention have been organized: (a) the development of Tutored Learning Projects, internship periods, Final Degree Projects, Master's Thesis and Doctoral Theses; (b) the development of an interclassroom seminar on Formative and Shared Evaluation, as a permanent training activity for teachers in all stages of education, from early childhood education to university. We have obtained as a result advantages, disadvantages and proposals for improvement for later courses from the point of view of student learning, teacher teaching and the teaching and learning process itself.

Keywords: Formative assessment; Shared assessment; Knowledge transfer; Inservice teacher education; Pe-service teacher education.

*Contacto: cristina.pascual@uva.es

issn: 1989-0397

www.rinace.net/riee/

https://revistas.uam.es/riee
Recibido: $\quad 17$ de enero de 2019

$1^{\text {a }}$ Evaluación: 04 de febrero de 2019

$2^{\text {a }}$ Evaluación: 25 de ferero d 2019

Aceptado: $\quad 01$ de marzo de 2019 


\section{Introducción}

El Proyecto de Innovación Docente (PID) que presentamos se desarrolla en el ámbito de la Universidad de Valladolid, en los campus de Segovia, Valladolid y Soria. Desde la Formación Inicial del Profesorado (FIP) es necesario que planteemos la formación a futuros maestros y maestras en metodologías y formas de evaluación innovadoras. Para lograr este objetivo es necesario a su vez estar en contacto con la realidad escolar en la que se sumergirán dichos docentes.

En consecuencia, a través de este PID hemos desarrollado una transferencia de conocimiento en dos direcciones: desde la Universidad a los centros educativos y desde la realidad escolar a la formación universitaria. Para ello se han organizado dos líneas de intervención: (a) experiencias de formación en la FIP a través de la realidad concreta de los centros educativos: "Proyectos de Aprendizaje Tutorado" (PAT) que se desarrollan en los colegios; periodos de Prácticum, Trabajos Fin de Grado (TFG), Trabajos Fin de Máster (TFM) y Tesis Doctorales; (b) desarrollo de seminarios de Formación Permanente del Profesorado (FPP) con docentes de todas las etapas educativas desde Educación Infantil hasta Educación Universitaria.

La primera línea de intervención se lleva a cabo fundamentalmente en la FIP. Los alumnos en formación desarrollan competencias profesionales sobre la Evaluación Formativa y Compartida (EFyC) y llevará dichas propuestas de innovación a centros educativos a través de diferentes actividades prácticas y académicas antes señaladas. Ejemplo de esta intervención es la que se ha desarrollado en el anterior curso 2017/2018 con un TFG que se desarrolló en un aula de Educación Primaria, en concreto en el aula de Educación Física donde su autora realizó su periodo de Prácticum. Fernández (2018) obtuvo como resultados de su trabajo una gran fiabilidad y significatividad de su sistema de EFyC y las calificaciones obtenidas. De la misma manera, se desarrolló un TFM en el pasado curso 2017/2018, (Ayuso, 2018) el cual se centró en conocer la percepción del alumnado de FIP del Grado de Maestro en Educación Infantil sobre la aplicación de EFyC en algunas asignaturas del grado. Obtuvo como resultados una valoración alta de ventajas respecto al empleo de EFyC en la FIP, inconvenientes vinculados a la exigencia de implicación por parte del alumnado, una evolución muy satisfactoria lo largo de los 4 cursos analizados y una posibilidad de transferencia de conocimiento de estos sistemas de EFyC en la FIP a las etapas iniciales educativas en su futura docencia.

Estos dos ejemplos nos muestran la necesidad de unión entre la universidad y la escuela. La transferencia de conocimiento que se produce es recíproca, lo que nos lleva a la segunda línea de intervención: el desarrollo del seminario de Formación Permanente del Profesorado (FPP). Las dinámicas de EFyC que los participantes del seminario de FPP han desarrollado durante el curso 2017/2018 y en el actual curso, generan dinámicas de investigación-acción que pueden ser útiles para formar a futuros y futuras docentes. En este sentido toman especial relevancia las dos tesis doctorales que se están llevando a cabo vinculadas claramente al PID.

Por todo lo dicho hasta ahora, los objetivos principales del desarrollo del PID son los siguientes:

- Crear una red interdisciplinar de investigadores universitarios que imparten docencia en la FIP y de maestros que trabajen en centros de Educación Infantil, 
Primaria y Secundaria para desarrollar seminarios permanentes de investigaciónacción sobre evaluación formativa y compartida en educación.

- Conocer los sistemas de evaluación formativa y compartida que se desarrollan en colegios de Soria, Segovia, y Valladolid y ahondar en los beneficios que éstos suponen aportar en procesos colaborativos con la formación inicial del profesorado (PAT, Prácticum, Intervenciones puntuales en colegios, TFG, TFM y Tesis Doctorales).

- Realizar una transferencia de conocimiento entre escuela y universidad, mediante un seminario de formación permanente con maestros de todas las etapas educativas. Conocer así las prácticas más innovadoras y excelentes de EFyC.

- Realizar una transferencia de conocimiento desde la universidad al colegio : (1) mediante el aporte de ideas innovadoras por parte de los docentes universitarios implicados en el PID; (2) mediante las prácticas de los estudiantes que desarrollen sus prácticas profesionales en el centro así como estudiantes que vayan a desarrollar su Trabajo Final de Grado (TFG) y tesis sobre esta temática; así como en el desarrollo de seminarios de formación permanente e investigación-acción con el profesorado interesado en desarrollar este tipo de innovaciones.

- Elaborar documentos en los que se recojan técnicas e instrumentos y buenas prácticas de $\mathrm{EFyC}$ para que sirvan de objeto de aprendizaje y difusión de las mismas.

\section{Revisión de la literatura}

\subsection{Evaluación Formativa y Compartida en el desarrollo del Proyecto de Innovación Docente}

En la educación actual es necesario un cambio metodológico en consonancia con todos los elementos de la realidad educativa. Por tanto, cualquier innovación educativa que no vaya acompañada de la adecuada innovación en evaluación, fracasará (Barberá, 2003; Biggs, 2005; Bonsón y Benito, 2005; Knight, 2005; Zabalza, 2001).

La evaluación es una de las tareas principales del profesorado, pero para que aporte mejoras al sistema de aprendizaje y de la calidad docente ha de sufrir fuertes cambios por dos motivos: es necesario cambiar el objeto de la evaluación de los conocimientos más o menos académicos a las competencias y conocimientos básicos y aplicados más complejos; así como cambiar profundamente el papel que puede y debe jugar la evaluación (Hamodi, López-Pastor y López, 2015).

Según diferentes autores (López-Pastor, 2009; López-Pastor y Pérez-Pueyo, 2017; LópezPastor, Hamodi y López, 2016), la evaluación formativa se podría entender cómo todo proceso de evaluación con la finalidad de mejorar el proceso de Enseñanza-Aprendizaje (E-A) y por consiguiente, sus tres líneas de intervención: a) mejora del aprendizaje del alumnado para que aprenda más y mejore las evidencias del aprendizaje que genera; b) mejora del proceso de enseñanza del docente; c) mejora del proceso de E-A paulatinamente, corrigiendo los errores detectados y fomentando las vías de actuación que sí funcionan. Por otro lado, la evaluación compartida es definida por los mismos autores como aquella que implica al alumnado en los procesos de evaluación (mediante diálogos 
con el profesor, procesos de autoevaluación, evaluación entre iguales, evaluación compartida, etc.).

Existen numerosos autores y estudios que defienden la participación del alumnado en la evaluación formativa, para resumir las ventajas que encuentran tomaremos como referencia las que aclaran en diversos textos Hamodi, López y López-Pastor (2014) y López-Pastor (2008, 2009, 2012): a) mejora el aprendizaje; b) mejora la autonomía personal, la autorregulación y el pensamiento crítico y autocrítico; c) desarrolla la formación de personas responsables acordes con el desarrollo de una educación democrática; (d) mejora el clima del aula y la resolución de problemas de convivencia en el aula y/o centro.

\subsection{Evaluación Formativa y Compartida en la Formación Inicial del Profesorado}

Cañadas, Santos-Pastor y Castejón (2018) señalan que la EFyC toma especial relevancia en la FIP porque los docentes universitarios deben aplicar estrategias metodológicas y evaluativas que permitan afianzar las competencias claves durante la FIP y que puedan ser transferibles en su futuro como docentes. Dentro de la transferencia de conocimiento que se desarrolla desde la universidad a la escuela, la EFyC en la FIP es muy relevante en los procesos de E-A que desarrollan los alumnos, pues como hemos detallado anteriormente, tanto los PAT como periodos de Prácticum, TFG, TFM y Tesis Doctorales, sirven a los docentes en formación para aplicar y aprender de prácticas de $\mathrm{EFyC}$ en un contexto real.

En esta línea de intervención centrada en la FIP, los PAT tienen gran presencia a lo largo de todo el proceso de formación. Son muchos los profesores universitarios que en nuestro contexto desarrollan estas prácticas de aprendizaje en EFyC. Para aclarar la dinámica de desarrollo de los PAT, tomaremos como referencia los textos de Manrique-Arribas, López-Pastor y Real-Rubio (2010) y Martínez-Mínguez (2016), que los definen como una actividad grupal donde se ponen en juego diferentes competencias profesionales de los futuros docentes. En ambos textos se recoge que la elaboración de los PAT requiere: (1) un alto grado de trabajo; (2) revisiones con el tutor; y (3) trabajo continuado a través de feedback y mejoras. Pero a su vez los estudiantes lo consideran una actividad necesaria, satisfactoria y que aporta aprendizajes muy significativos. El desarrollo de trabajo de los PAT asegura una calidad y un aprendizaje mínimo tanto en el trabajo como en los propios participantes. Existen muchas otras experiencias que constatan la eficacia de la relación entre la FIP y la EFyC. Como es el caso de Barba-Martín y López-Pastor (2017), quienes presentan su experiencia con los PAT en el último curso del Grado de Maestro en Educación Infantil. Obtienen como resultados una valoración positiva tanto por parte del alumno como de los docentes. Además, los propios alumnos reflejan en los cuestionarios utilizados una autopercepción de las competencias adquiridas muy altas con este proceso.

En cuanto a los periodos de Prácticum podemos tomar como referencia el texto de Gallardo-Fuentes y Carter (2016), los cuales analizan la implementación de la EFyC en el periodo de prácticum en la FIP. Coincidimos con estos autores al considerar que la EF yC en el desarrollo del Prácticum permite a los alumnos desempeñar un papel activo y participativo, además de fomentar el aprendizaje continuo a través de sus prácticas docentes. Los participantes de este estudio valoran la claridad en torno al sistema de evaluación formativa y al feedback que se produce en todo el proceso. Una experiencia similar es la que exponen Gallardo-Fuentes, López-Pastor y Carter-Tuhillier (2018), en 
la que obtienen como resultado que tras el desarrollo de prácticas de EFyC en la FIP sus valoraciones de autopercepción en la adquisición de competencias son más altas que antes de cursarlo y consideran que hay más ventajas que inconvenientes en la utilización de estas prácticas.

Este trabajo continuo con el alumnado se produce de igual manera en otras de las actividades de esta primera línea de intervención del PID. En el caso del TFG que se ha vinculado al PID durante el curso 2017/2018, desarrolló una experiencia real de EFyC en un aula de Educación Primaria, obteniendo como resultado un sistema de evaluación muy significativo para el alumnado. Sobre el aprendizaje en esta práctica podemos afirmar que tiene una doble vía: (a) por una parte, la maestra en prácticas aplica sus sistemas de $\mathrm{EFyC}$ en un contexto real, adaptándose al alumnado de Primaria; (b) además, la maestra en prácticas también aprende sobre EFyC en la elaboración de su propio TFG. De una manera muy similar se produce la elaboración de los TFM y Tesis Doctorales. En nuestro caso durante el curso 2017/2018 se elaboró un TFM vinculado al PID y dieron comienzo dos Tesis Doctorales.

La utilización de sistemas de $\mathrm{EFyC}$ en FIP nos permite que el alumnado tome conciencia de su nivel de competencias y pueda transferir estas prácticas en su docencia futura. Por otro lado, el profesorado de FIP puede aprender a través de los sistemas de EFyC, dado que también están enfocados a mejorar el proceso de E-A que se lleva a cabo en la asignatura y a desarrollar su calidad docente (López-Pastor, 2009). Existe una relación entre las prácticas de EFyC que desarrollan en sus asignaturas, con la evolución y los resultados observados en cada curso escolar, pudiendo así orientar su docencia hacia los aspectos que funcionan y buscar alternativas a los aspectos que no son tan fructíferos (Hamodi, López-Pastor y López, 2017; Manrique-Arribas, Vallés y Gea, 2012).

\subsection{Evaluación Formativa y Compartida en la Formación Permanente del Profesorado}

La segunda línea de intervención se lleva a cabo a través del seminario de FPP que se desarrolla sobre esta temática en la Facultad de Educación de Segovia, denominado "Seminario de EFyC en Educación". Se ha desarrollado durante el curso 2017/2018, y se sigue desarrollando en el curso actual 2018/2019. En el seminario hemos desarrollado diferentes actividades de aprendizaje a partir de la metodología de Investigación-Acción (I-A). Esta metodología de trabajo permite desarrollar procesos cíclicos de aprendizaje con las siguientes fases: 1) planificar; 2) actuar; 3) observar; 4) analizar lo ocurrido (Kemmis y McTaggart, 1998; Latorre, 2003).

Esta modalidad de FPP mediante la I-A tiene una larga trayectoria en el área de Educación Física con resultados muy satisfactorios durante los últimos 20 años. Ejemplo de ellos son también experiencias como la que expone Barba-Martín, Barba y Martínez (2016), Córdoba et al. (2016), López-Pastor et al. (2016) y López, Monjas y Manrique (2011). Además del desarrollo de la I-A en su práctica docente, también se produce aprendizaje colaborativo entre docentes, al poner en común las reflexiones y experiencias de $\mathrm{EFyC}$ que cada docente desarrolla en su aula. Por tanto, la transferencia de conocimiento se produce a partir de las dinámicas de I-A que surgen en el seminario y que pueden ser útiles para los docentes de manera que: (1) los docentes de Educación Infantil, Educación Primaria y Educación Secundaria aprenden de las prácticas de sus propios compañeros y mejoran su práctica docente a partir de las dinámicas colaborativas de I-A; (2) los docentes 
de Educación Universitaria, además de aprender de las prácticas de sus compañeros, se acercan a la realidad del aula, pudiendo mejorar en consecuencia la docencia que imparten en la FIP.

Tras el desarrollo del seminario durante el curso 2017/2018, podemos terminar con esta línea de intervención señalando las ventajas observadas en consonancia con López-Pastor et al. (2016): (1) La participación en grupos de trabajo de I-A supone un fuerte estímulo y ayuda en el desarrollo profesional docente; (2) parece ser un elemento importante en la mejora de la calidad docente del profesorado implicado en el mismo, así como un fuerte apoyo para ir solucionando problemas que surgen en la práctica educativa; (3) favorece el desarrollo profesional docente y la mejora de la calidad educativa.

Los objetivos de investigación que nos proponemos son:

- Evaluar el funcionamiento de la doble línea de intervención del PID durante el curso 2017/2018 analizando las actividades desarrolladas.

- Analizar las ventajas, inconvenientes y propuestas de mejora que los participantes del PID han manifestado sobre las prácticas de EFyC que han desarrollado.

\section{Método}

\subsection{Enfoque metodológico}

Consideramos el enfoque metodológico desde la perspectiva del estudio de caso, en concreto, del estudio de caso evaluativo (Pérez, 1994), entendida como la metodología que nos permite comprender de manera exhaustiva un tema en concreto para generar conocimientos e informar de ese caso específico, para describir y explicar una realidad para poder emitir juicios sobre la misma (Álvarez y San Fabián, 2012; Simons, 2011; Stake, 2010). Tomando estos mismos autores de referencia, entendemos que el estudio de caso se adecúa a la situación de investigación que hemos y estamos desarrollando. A través de la descripción, documentación e interpretación de lo que sucede en un escenario real. Este método es flexible, no es dependiente del tiempo ni está limitado por ningún método, por lo tanto, se adecúa al desarrollo del PID a lo largo de los cursos escolares (2017/2018 y 2018/2019). A través del razonamiento inductivo del estudio de caso, partiremos de lo más particular a lo más general, conociendo cada contexto real que se vincule al PID, para llegar a unas generalizaciones que puedan surgir de las aportaciones de los participantes.

\subsection{Categorías de análisis}

Cómo hemos dividido a lo largo del capítulo la doble vía de intervención dentro del PID, condicionará el estudio de caso y las categorías de análisis de igual forma. Por un lado, analizaremos la primera línea de intervención en la FIP mediante los PAT, periodos de Prácticum, TFG, TFM y Tesis Doctorales que se desarrollaron; y por otro lado, la segunda línea de intervención con el seminario de FPP sobre EFyC. Dentro de estas categorías analizaremos tres subcategorías iguales para ambas vías: ventajas que se han encontrado en las prácticas, inconvenientes y propuestas de mejora. Las categorías de análisis quedarán establecidas de la siguiente manera como se puede ver en el cuadro 1. 
Cuadro 1. Categorías y subcategorías de análisis

\begin{tabular}{|c|c|}
\hline CATEGORÍAS DE ANÁLISIS & SUBCATEGORÍAS DE ANÁLISIS \\
\hline \multirow{3}{*}{$\begin{array}{l}\text { Línea de intervención en la FIP (PAT, } \\
\text { Prácticum, TFG, TFM y Tesis) }\end{array}$} & Ventajas \\
\hline & Inconvenientes \\
\hline & Propuestas de mejora \\
\hline \multirow{3}{*}{$\begin{array}{l}\text { Línea de intervención en la FPP (Seminario de } \\
\text { FPP sobre EFyC) }\end{array}$} & Ventajas \\
\hline & Inconvenientes \\
\hline & Propuestas de mejora \\
\hline
\end{tabular}

Fuente: Elaboración propia.

\subsection{Participantes}

El PID se ha desarrollado durante el curso 2017/2018 con un total de 261 participantes: 205 alumnos y alumnas que han desarrollado sus Proyectos de Aprendizaje Tutorado en los colegios con prácticas de $\mathrm{EFyC}$; 30 alumnos que han desarrollado prácticas de EFyC en sus Prácticum; 1 alumna de Trabajo Fin de Grado; 1 alumna de Trabajo Fin de Máster; 2 doctorandas desarrollando su tesis sobre $\mathrm{EFyC} ; 22$ docentes de todas las etapas educativas, participantes en el seminario de formación permanente. Detallamos estos datos en el cuadro 2.

Cuadro 2. Participantes PID EFyC curso 2017/2018

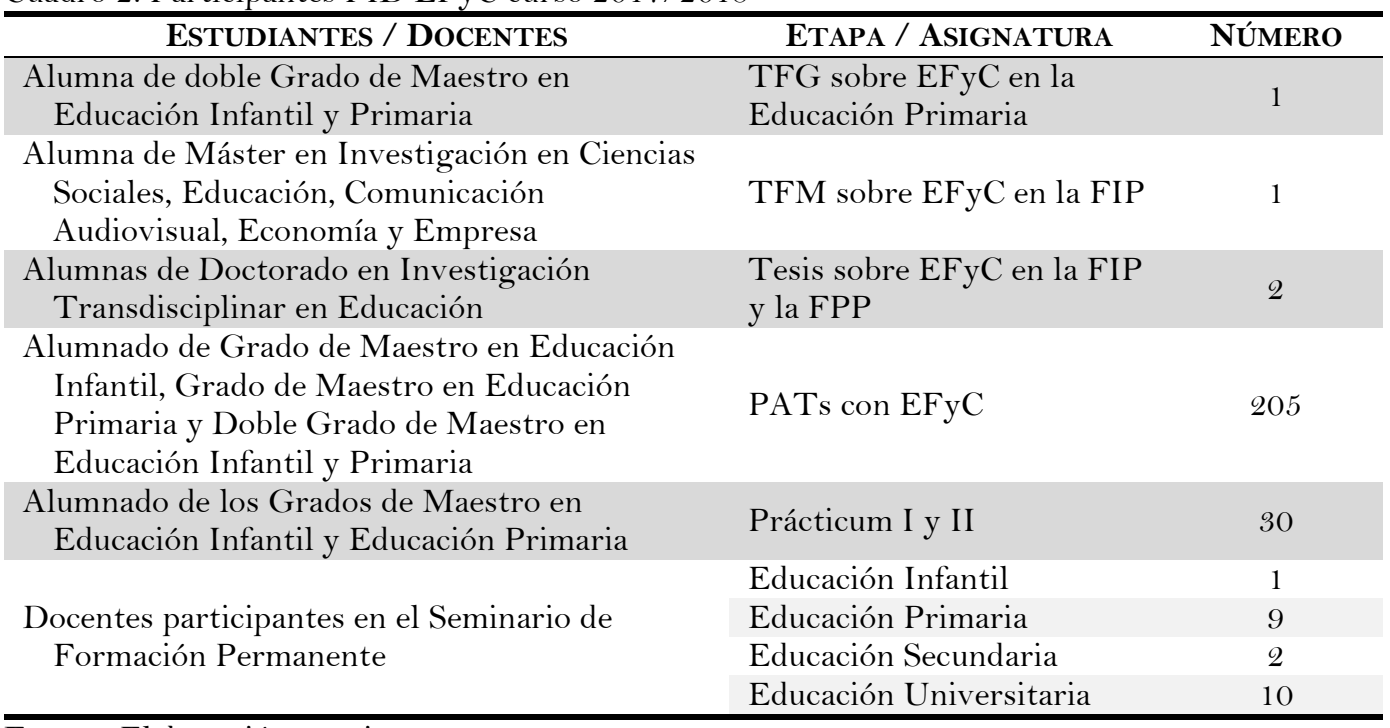

Fuente: Elaboración propia.

\subsection{Instrumentos de obtención de información}

Los instrumentos de obtención de información que hemos utilizado han sido dos principalmente:

- Actas de reuniones del seminario: durante el curso 2017/2018 el desarrollo del seminario ha quedado recogido en las actas de sesión que se han ido elaborando después de cada reunión. Estas doce actas contienen datos relativos a la asistencia de los participantes a cada sesión, los temas de debate que surgen, las dudas y reflexiones expuestas, así como los acuerdos logrados o el día concretado de la próxima reunión. Gracias al análisis de las actas podemos comprobar cuál ha sido la evolución de sus participantes, así como extraer los resultados de ventajas, 
inconvenientes y propuestas de mejora diferenciando entre la etapa obligatoria (infantil/primaria y secundaria) y la etapa universitaria.

- "Informes de $\mathrm{EFyC}$ " desarrollados por los participantes del seminario: durante el curso 2017/2018 los participantes del seminario de formación permanente elaboraron informes de buenas prácticas docentes sobre las prácticas desarrolladas en su aula. Estos informes están establecidos por la Red de Evaluación Formativa y Compartida, la cual cuenta con una larga trayectoria desde el año 2005 y sirve como grupo de trabajo de referencia en innovación e investigación sobre $\mathrm{EFyC}$ (Hamodi et. al, 2014). Existen tres tipos de informes con una estructura determinada por la Red de Evaluación Formativa y Compartida: (a) etapa no universitaria; (b) etapa universitaria sencillo, sin cuestionarios de investigación; (c) etapa universitaria complejo, con resultados de cuestionarios de investigación. Durante la primera parte del curso (de septiembre hasta enero/febrero aproximadamente), se realizó la parte de planificación de cada proceso de EFyC. En esta parte se detallaron aspectos relativos al contexto donde desarrollan su docencia, las actividades de $\mathrm{EFyC}$ o los instrumentos a utilizar. En la segunda parte del curso (de febrero a junio), se fue completando el informe, plasmando los resultados de cada caso, analizando ventajas e inconvenientes, así como las propuestas de mejora para seguir trabajando y mejorando. A lo largo del curso los informes se han corregido de manera progresiva por los coordinadores de dicho seminario, a través de las reuniones presenciales y el correo electrónico. Al final del curso, cada informe queda completo con las experiencias de cada docente, para compartir dichos procesos con los demás miembros de la Red.

Cuadro 3. Codificación de documentos analizados

\begin{tabular}{|c|c|c|}
\hline DOCUMENTOS ANALIZADOS & \multicolumn{2}{|c|}{ LISTADO DE DOCUMENTOS Y CÓDIGO } \\
\hline \multirow{6}{*}{$\begin{array}{l}\text { Actas de seminarios FPP } \\
\text { curso } 2017 / 2018(12)\end{array}$} & Acta $0-$ AoSEFyCSG & Acta $6-\mathrm{A} 6 \mathrm{SEF} y \mathrm{CSG}$ \\
\hline & Acta $1-$ A1SEFyCSG & Acta 7 - A7SEFyCSG \\
\hline & Acta $2-\mathrm{A} 2 \mathrm{SEFyCSG}$ & Acta 8 - A8SEFyCSG \\
\hline & Acta 3 - A3SEFyCSG & Acta 9 - A9SEFyCSG \\
\hline & Acta 4-A4SEFyCSG & Acta $10-$ A 10 SEFyCSG \\
\hline & Acta 5 - A5SEFyCSG & Acta $11-$ A 11 SEFyCSG \\
\hline \multirow{6}{*}{$\begin{array}{l}\text { Informes de evaluación de } \\
\text { los participantes del PID }\end{array}$} & $\begin{array}{c}\text { Informe Buenas Prácticas } \\
\qquad 1-\text { IBP } 1\end{array}$ & $\begin{array}{c}\text { Informe Buenas Prácticas } 7 \\
\text { - IBP7 }\end{array}$ \\
\hline & $\begin{array}{c}\text { Informe Buenas Prácticas } \\
\qquad 2-\mathrm{IBP}_{2}\end{array}$ & $\begin{array}{c}\text { Informe Buenas Prácticas } 8 \\
-\mathrm{IBP} 8\end{array}$ \\
\hline & $\begin{array}{c}\text { Informe Buenas Prácticas } \\
3-\mathrm{IBP}_{3}\end{array}$ & $\begin{array}{c}\text { Informe Buenas Prácticas } 9 \\
-\mathrm{IBP} 9\end{array}$ \\
\hline & $\begin{array}{c}\text { Informe Buenas Prácticas } \\
4-\mathrm{IBP}_{4}\end{array}$ & $\begin{array}{c}\text { Informe Buenas Prácticas } 10 \\
- \text { IBP10 }\end{array}$ \\
\hline & $\begin{array}{c}\text { Informe Buenas Prácticas } \\
5-\text { IBP } 5\end{array}$ & $\begin{array}{c}\text { Informe Buenas Prácticas } 11 \\
\text { - IBP } 11\end{array}$ \\
\hline & $\begin{array}{c}\text { Informe Buenas Prácticas } \\
6-\mathrm{IBP} 6\end{array}$ & $\begin{array}{c}\text { Informe Buenas Prácticas } 12 \\
- \text { IBP } 12\end{array}$ \\
\hline
\end{tabular}

Fuente: Elaboración propia.

\subsection{Análisis de datos}

El análisis de datos lo hemos realizado mediante una doble vía: 1) por un lado, la transcripción a lo largo del curso 2017/2018 de las actas de los seminarios de FPP; 2) por 
otro lado, el análisis de los informes de evaluación que cada participante del PID ha remitido a los coordinadores. A través de la codificación que presentamos en el cuadro 3 hemos analizado dichas actas e informes. Se ha optado por no utilizar programas informáticos para este análisis. Para la obtención de resultados hemos analizado dichos datos con las categorías y subcategorías establecidas en el cuadro 1. Los datos recogidos del desarrollo del PID en el curso 2017/2018 los analizamos a través de las dos categorías principales y los dividimos en las subcategorías en los cuadros 4 y 5.

\section{Resultados}

Comenzaremos mostrando los resultados de la línea de intervención en la FIP durante el curso 2017/2018. Al analizar los datos de los informes de EFyC desarrollados por los 22 docentes participantes en el seminario, hemos recogido en el cuadro 3 las ventajas, inconvenientes y propuestas de mejora de los mismos que han reflejado en dichos informes.

Cuadro 4. Resultados de la línea de intervención en la FIP. Ventajas, inconvenientes y propuestas de mejora

LíneA DE INTERVENCIÓN EN LA FORMACIÓN INICIAL DEL PROFESORADO

\section{Ventajas}

Buen clima de trabajo en el aula, elevada motivación en las actividades realizadas con escolares en el Campus Universitario ya que pueden diseñar sus propuestas, implementarlas y evaluarlas junto a con los maestros implicados en las actividades.

Tasa de éxito elevada. Buena calidad en muchos de los PAT realizados y buena preparación en gran parte de los grupos.

Los alumnos consideran que se han visto favorecidos en la adquisición de competencias profesionales que se les exige en su titulación, indicando que se ha conseguido entre mucho y bastante.

Se favorece la puesta en común y el debate de ideas. En consecuencia, se reafirma el aprendizaje colaborativo entre iguales y el establecimiento de conclusiones mucho más válidas.

Hacer copartícipe al alumno de su propio aprendizaje y del proceso de evaluación. Desarrollar la capacidad de reflexión del alumno para generar un futuro docente reflexivo y comprometido con la cultura de la EFyC.

Alta implicación del alumno tanto en el trabajo y desarrollo de los PAT como en la elección de la temática a trabajar.

\begin{tabular}{lc}
\hline \multicolumn{3}{c}{ Inconvenientes } & Propuestas de mejora \\
\hline $\begin{array}{l}\text { Grupos de PAT con poca iniciativa y } \\
\text { demanda de información y guía para el }\end{array}$ & $\begin{array}{c}\text { Hacer más explícitas las tareas y los } \\
\text { criterios acordados desde el inicio de las } \\
\text { desarrollo de actividades previstas, } \\
\text { actividades. }\end{array}$ \\
$\begin{array}{l}\text { sobretodo en la organización de } \\
\text { documentos. }\end{array}$ & $\begin{array}{c}\text { Marcar con más antelación los tiempos y } \\
\text { Los alumnos de PAT solicitan más tiempo }\end{array}$ \\
$\begin{array}{l}\text { y muestran cierto agobio con las tareas } \\
\text { solicitadas. }\end{array}$ & $\begin{array}{c}\text { tareas que se tienen que realizar. Se } \\
\text { puede plantear con mayor selección de }\end{array}$ \\
$\begin{array}{c}\text { Lostividades que tienen que entregar. } \\
\text { mejorar los documentos de PAT una vez }\end{array}$ & $\begin{array}{c}\text { Se puede dar la posibilidad de mejorar en } \\
\text { ellas, en función de la calidad de las }\end{array}$ \\
revisados en algunas actividades. & mismas. \\
Algunos grupos se preparan el trabajo de & Utilizar de forma sistemática la plantilla \\
PAT de forma deficiente. & de autoevaluación y autocalificación de
\end{tabular}


Gran carga de trabajo para el alumnado, expresan que dedican muchas horas para elaborar las actividades obligatorias que son exigidas para completar la evaluación continua y formativa.

Acumulación de trabajo en algunas semanas concretas donde coincide la entrega de un gran número de trabajos.

Desconocimiento en ocasiones del grado de aprendizaje que los alumnos tienen sobre los contenidos que se abordan cada semana de docencia.

La coevaluación no ha sido siempre efectiva tras analizar los datos aportados por los alumnos.

La autoevaluación no siempre se utiliza como se debiera, con rigor y ajustándose a la realidad de cada alumno.

La comprensión de las rúbricas por parte de algunos alumnos. los PAT, ofrece una orientación importante al alumnado.

Reducir el número de artículos o documentos sobre los que tienen que hacer el trabajo, así como aligerar el contenido de las fichas de sesiones prácticas para que no se convierta en un trabajo monótono y les desmotive.

Permitir posponer las entregas a los grupos implicados en el PAT.

Realizar pruebas on line de resolución inmediata que contribuyan, tanto al profesor como al alumno a tener una mejor idea de los contenidos más difíciles de aprender y los que no hay problema.

Concienciar al alumnado de la necesidad de entender la coevaluación como una evaluación entre iguales con rigor.

Mentalizar al alumno ante la evaluación crítica de su propio trabajo, asumiendo posibles errores y viendo las soluciones que se pueden aplicar a los mismos.

Explicación exhaustiva de las mismas al comienzo de la asignatura para que perciban la importancia que tienen y el grado de consecución.

Fuente: Elaboración propia.

Podemos observar que las ventajas que destacaron los docentes se orientan hacia la participación del alumnado, la tasa de éxito que obtuvieron y su desarrollo de la autocrítica y el rigor profesional como futuros docentes. Unido a estas ventajas señalan la motivación del alumnado por aprender más y por el trabajo en el proceso de E-A. Al hacer copartícipe al alumnado de su propio aprendizaje y del proceso de evaluación los docentes encuentran también una alta implicación en sus alumnos. Como señala uno de los participantes en su informe de evaluación:

Las ayudas que reciben del profesor han sido muy valoradas, probablemente por los comentarios que se les realiza al comentar cada una de las tareas y actividades que tienen que realizar. Sin embargo, aprecian más las de sus propios compañeros, lo cual resulta atractivo por su componente motivacional y porque las aclaraciones que éstos emiten suelen ser más perdurables y significativas en el tiempo. (IBP5, página 8)

En cuanto a los inconvenientes, podemos comprobar que valoran las opiniones del alumnado como su manifestación hacia la carga de trabajo y proponen como solución la adaptación de dicho trabajo al ritmo del alumnado. Varios de los inconvenientes que han encontrado los docentes hacen referencia al proceso de elaboración de PAT, como, por ejemplo: poca iniciativa del alumnado, demanda de mayor tiempo de elaboración, preparación deficiente... A todos estos inconvenientes se proponen soluciones similares: hacer más explícitos los criterios de evaluación con diferentes instrumentos de evaluación formativa y compartida. De esta manera los alumnos serán más conscientes de las "reglas 
de juego" y se podrá dar un paso más: que las asuman como propias si participan en el establecimiento de las mismas.

En el cuadro 4 vemos los resultados de la otra línea de intervención, referente a la FPP durante el curso 2017/2018. Tras analizar los datos recogidos en las actas de los seminarios de formación permanente, los docentes participantes destacaron estas ventajas, inconvenientes y propuestas de mejora.

Podemos comprobar algunas similitudes entre la tabla 3 y 4, ya que, aunque sean líneas de intervención diferentes, algunos de los docentes se han encontrado con inconvenientes similares, como puede ser el caso de la incomprensión de las rúbricas como instrumento de evaluación., o la desconfianza del alumnado hacia el sistema de EFyC cuando se desarrolla por primera vez. Ante estas situaciones, los docentes proponen propuestas de mejora similares: explicar con claridad el cambio educativo y los nuevos instrumentos de evaluación que se van a utilizar, así como dar valor a los aprendizajes que se van a producir para atraer a los alumnos hacia el cambio. Una de las participantes al seminario de FPP aporta como reflexión en su informe de evaluación:

\section{El manejo del Diario al principio de la práctica origina posturas discordantes entre algunos estudiantes puesto que tienen que hacer una tarea que, tradicionalmente, no se relacionan con el área. Sin embargo, una vez que han entendido la rutina y el proceso del manejo de estos instrumentos, deja de ocasionar cualquier tipo de rechazo por su parte. (IBP2, página 6)}

También observamos, como ventaja a destacar, que tanto alumnado como docentes le restan importancia a la calificación, para dar más importancia y poner en valor su aprendizaje en el desarrollo en el proceso de E-A. Valoran también como aspecto muy positivo que los alumnos a través de la $\mathrm{EFyC}$ son conscientes de su ritmo de aprendizaje, de los aspectos que han aprendido y lo que queda por aprender.

Otra de las observaciones que destacamos tras el análisis de las actas es que los maestros valoran el poder reconducir su práctica docente a lo largo del proceso gracias a la EFyC. Durante el seminario gracias al aprendizaje colaborativo, al feedback que reciben de los coordinadores y a sus propias observaciones en su praxis mejoran su práctica docente, reconducen el proceso y, en consecuencia, ayudan a mejorar el aprendizaje del alumnado.

Cuadro 5. Resultados de la línea de intervención en la FPP. Ventajas, inconvenientes y propuestas de mejora

Línea de Intervención en la Formación PERManente del Profesorado (ETAPA OBLIGATORIA Y ETAPA UNIVERSITARIA) Ventajas

Mejora de la praxis docente de manera constante, adecuándose al ritmo de los alumnos y mejora de las prácticas que no funcionaban.

Mejora de la atención a la diversidad del alumnado en el aula, enseñanza más individualizada y con mejoras en la relación docente-alumno.

Gracias al feedback continuo e individualizado con el alumnado mejora su participación, sus diálogos son más justos y consecuentes con su trabajo y tareas.

El proceso de enseñanza mejora porque el maestro puede reconducir el proceso a lo largo de todo el curso, resolviendo problemas y buscando alternativas a lo que no funciona en el aula, así como fomentar las prácticas de éxito.

Mejora del aprendizaje colaborativo entre docentes, tanto en el desarrollo del seminario como en su práctica docente.

Los alumnos a través de la EFyC tienen consciencia de su ritmo de aprendizaje, el punto del proceso en el que se encuentran y cómo pueden mejorar. 
Incremento de la participación del alumnado al entender que su aportación es relevante y mejora su capacidad de responsabilidad, reflexión y autocrítica.

La EFyC aporta transparencia al proceso, se conocen los instrumentos que se van a utilizar, la carga de trabajo del alumnado y del docente.

Se resta importancia a la calificación y se da más importancia al aprendizaje durante todo el proceso.

\begin{tabular}{|c|c|}
\hline Inconvenientes & Propuestas de mejora \\
\hline $\begin{array}{l}\text { Desconfianza del alumnado hacia el } \\
\text { sistema de evaluación por } \\
\text { desconocimiento. }\end{array}$ & $\begin{array}{l}\text { Explicar con claridad el cambio educativo, } \\
\text { demostrar beneficios del sistema en otros } \\
\text { grupos y dar valor a los aprendizajes de } \\
\text { mayor calidad que se van a producir. }\end{array}$ \\
\hline $\begin{array}{l}\text { Mayor carga de trabajo docente por la } \\
\text { cantidad de instrumentos a diseñar } \\
\text { al comienzo del proceso de EFyC }\end{array}$ & $\begin{array}{l}\text { Desarrollar instrumentos flexibles y } \\
\text { adaptables a la evaluación de situaciones } \\
\text { similares. Llevar a cabo metodologías más } \\
\text { acordes con la evaluación que incluyan } \\
\text { instrumentos que abarquen mas información } \\
\text { y recursos para este sistema. }\end{array}$ \\
\hline $\begin{array}{l}\text { La evaluación entre pares no ha sido } \\
\text { siempre veraz o rigurosa tras } \\
\text { analizar los datos de los alumnos, ya } \\
\text { que, en ocasiones, al evaluar a sus } \\
\text { compañeros } \\
\text { cohibiciones o evalúa con poco } \\
\text { rigor. }\end{array}$ & $\begin{array}{l}\text { Hacer ver al alumnado la necesidad e } \\
\text { importancia de realizar la evaluación entre } \\
\text { iguales con rigor y conciencia, para que esa } \\
\text { información pueda ser útil para el proceso de } \\
\text { E-A y la mejora de todos. }\end{array}$ \\
\hline $\begin{array}{l}\text { Incomprensión de las rúbricas por una } \\
\text { parte del alumnado }\end{array}$ & $\begin{array}{l}\text { Llevar a cabo explicaciones más exhaustivas } \\
\text { justo antes de su utilización, para que } \\
\text { comprendan mejor el significado de cada una } \\
\text { de sus categorías. }\end{array}$ \\
\hline
\end{tabular}

Fuente: Elaboración propia.

\section{Discusión y conclusiones}

Organizamos la discusión de resultados en función del sistema de categorías establecido. Por lo tanto, la presentaremos acorde a sus respectivas líneas de intervención: Formación Inicial del Profesorado (FIP) y Formación Permanente del Profesorado (FPP).

Los participantes que forman parte de la línea de intervención de la FIP consideran que hay más ventajas que inconvenientes en sus prácticas de $\mathrm{EFyC}$, aspecto que podemos ver en investigaciones similares (Gallardo-Fuentes et al., 2018; Romero-Martín, CastejónOliva, López-Pastor y Fraile-Aranda, 2017). Los participantes destacan que gracias a estas prácticas se fomenta un clima de trabajo óptimo, la motivación por diseñar propuestas e implementarlas en el aula es altísima, lo que conlleva a una tasa de éxito elevada, un trabajo muy enriquecedor y un aprendizaje significativo que los alumnos valoran (Romero-Martín, Castejón-Oliva y López-Pastor, 2015).

En cuanto a la adquisición de competencias, los participantes valoran como aspecto positivo la alta adquisición de competencias docentes y que estas prácticas favorecen el aprendizaje colaborativo, la puesta en común y el debate de ideas (Gallardo-Fuentes et al., 2018; López-Pastor, Pérez-Pueyo, Barba y Lorente, 2016). Si bien es cierto, consideramos que este aspecto tendríamos que investigarlo en profundidad atendiendo a qué competencias específicas se desarrollan en cada propuesta a desarrollar dentro de esta 
línea de intervención. Fomentar la participación del alumnado en el proceso de evaluación aporta más ventajas muy significativas, como el fomento de la capacidad reflexiva y crítica; además, vivirlo como alumnos durante su FIP aumenta las posibilidades de que lo utilicen y trasfieran en el futuro, cuando sean maestros (Romero-Martín, Asún y Chivite, 2016).

En cuanto a los inconvenientes de esta línea de intervención, podemos encontrarnos con aspectos que no se dan en la totalidad de alumnos. Como es el caso de la poca iniciativa a la hora de realizar los PAT, la solicitud de ampliación de plazos de entrega o la acumulación de trabajo. Solventar estos inconvenientes a partir de los resultados observados será una tarea que los docentes universitarios realizarán poniendo en práctica las propuestas de mejora sintetizadas de su proceso de EFyC (Martínez-Mínguez, 2016). Del mismo modo, gracias a lo observado durante el curso 2017/2018 los docentes que imparten docencia en la FIP aportan propuestas de mejora como desarrollar más procesos de feedback y la información que se aporta al principio de la asignatura correspondiente para facilitar la comprensión y el desarrollo del trabajo.

Respecto a la segunda línea de intervención del PID, referente a la FPP, podemos constatar que los participantes lo consideran un éxito. Durante el curso 2017/2018, 22 profesores de todas las etapas educativas participaron en el seminario, mientras que el presente curso son 24 los participantes. Muchos de ellos continúan desde el pasado curso.

A través de los procesos de investigación-acción los maestros ha mejorado su práctica educativa, partiendo de las necesidades de su alumnado (Barba-Martín et al., 2016; Hamodi et al., 2014). El éxito de este seminario de aprendizaje sobre EFyC recae en el aprendizaje colaborativo que se produce entre los participantes del mismo, aspecto que señalan como ventajas los participantes de nuestro PID y de otras experiencias como la de López-Pastor, Castejón, Sicilia-Camacho, Navarro y Webb (2011).

Otra de las ventajas es que los participantes del seminario pueden reconducir el proceso de E-A que estén llevando a cabo en su aula. Con esta reconducción del proceso, los docentes se adaptan a las necesidades de su grupo-clase, atienden a la diversidad del aula, respetan el ritmo de aprendizaje de los alumnos y favorecen la orientación del proceso hacia el aprendizaje (Barba-Martín, Barba y Martínez, 2016; López-Pastor et al., 2016).

Para finalizar con las limitaciones de esta vía de intervención, los participantes del seminario expusieron los inconvenientes con los que se habían encontrado en cada informe de evaluación. Algunos de ellos coinciden, como la incomprensión por parte del alumnado de algunos instrumentos de $\mathrm{EFyC}$ o la desconfianza del alumnado hacia un nuevo sistema de evaluación desconocido. Ante esta situación podemos afirmar que gracias a los ciclos de I-A que cada docente realiza en su aula estos inconvenientes se van solucionando progresivamente (López-Pastor et al., 2016). Muchos de ellos son comunes a todas las etapas educativas, desde la temprana Educación Infantil hasta la Educación Universitaria. Coincidiendo así estas limitaciones con algunas de las encontradas en la línea de intervención de la FIP y, por tanto, podemos considerarlo un punto de encuentro dentro de la transferencia de conocimiento de la universidad a la escuela y viceversa. Los objetivos generales han sido cumplidos. El funcionamiento del PID durante el curso 2017/2018 ha sido muy positivo. En las dos líneas de intervención se han realizado actividades de EFyC, lo que ha permitido reconducir la práctica docente y el proceso de E-A hacia la mejora continua en todas ellas. 
En cuanto al segundo objetivo, realizamos un balance muy positivo tras analizar las ventajas, inconvenientes y propuestas de mejora que los participantes del PID han manifestado. El alumnado de FIP ha desarrollado sus PAT, Prácticum, TFG, TFM y Tesis Doctorales vinculadas a la $\mathrm{EFyC}$ en un contexto real, fomentando la adquisición de competencias y reflexión autocrítica con su docencia gracias al contacto con la realidad de los centros educativos. Los participantes de la FPP aportan más ventajas que inconvenientes sobre el desarrollo de prácticas de EFyC en sus aulas y, además, a todos los inconvenientes que plantean buscan una propuesta de mejora para reconducir su práctica. Esto nos indica que los sistemas de $\mathrm{EFyC}$ suelen favorecer la orientación hacia el aprendizaje, respetar el ritmo de desarrollo y aprendizaje de los alumnos y atender a la diversidad del aula. También es importante señalar que todas las prácticas de éxito que los docentes de etapas obligatorias exponen en el seminario sirven a los docentes universitarios de FIP para acercar a sus alumnos a la realidad actual de las aulas, y así aprendizajes reales y de utilidad para su futuro como docentes.

Este trabajo puede ser de interés para los docentes de todas las etapas educativas en las que estén desarrollando prácticas de $\mathrm{EFyC}$ y quieran conocer otras prácticas de éxito o bien quieran iniciarse en la temática. También puede resultar de utilidad para el profesorado universitario de FIP interesado en este tipo de evaluación. A priori es posible que haya pocas experiencias que vinculen universidad y escuela de esta forma.

Como prospectiva de trabajo compararemos los resultados de la realización del seminario y de las prácticas docentes en la FIP vinculadas al PID tanto en el primer curso 2017/2018 con el presente 2018/2019, para ver la evolución de los aspectos desarrollados. Además, será interesante ver cómo se amplía la línea de formación del seminario en el resto de provincias del PID (Soria y Valladolid). La última línea de trabajo consistirá en comprobar la evolución de los TFG y TFM de este curso 2018/2019 y las dos Tesis Doctorales vinculadas directamente al PID.

\section{Referencias}

Álvarez, C. y San Fabián J. L. (2012). La elección del estudio de caso en investigación educativa. Gazeta de Antropología, 28(1).

Ayuso, N. (2018). Percepción del alumnado del grado de Educación Infantil sobre los sistemas de Evaluación Formativa y Compartida vividos en la Facultad de Segovia. Trabajo Fin de Máster. Universidad de Valladolid: Facultad de Educación de Segovia.

Barba-Martín, R. A., Barba, J. J. y Martínez Scott, S. (2016). La formación continua colaborativa a través de la investigación-acción. Una forma de cambiar las prácticas de aula. Contextos Educativos, 19, 161-175. https://doi.org/10.18172/con.2769

Barba-Martín, R. y López-Pastor, V. (2017). Evaluación formativa y compartida en los procesos de trabajo tutorado, un ejemplo de buena práctica. Revista Infancia, Educación y Aprendizaje, 3(2), 66-70. https://doi.org/10.22370/ieya.2017.3.2.701

Barberà, E. (2003). Estado y tendencias de la evaluación en Educación Superior. Revista de la Red Estatal de Docencia Universitaria, 3(2), 94-99.

Biggs, J. (2005). Calidad del aprendizaje universitario. Madrid: Narcea. 
Bonsón, M. y Benito, Á. (2005). Evaluación y aprendizaje. En Á. Benito y A. Cruz (Coords.), Nuevas claves para la docencia universitaria en el Espacio Europeo de Educación Superior (pp. 87-100). Madrid: Narcea.

Cañadas, L., Santos-Pastor, M. L. y Castejón, F. J. (2018). Desarrollo de competencias Docentes en la Formación Inicial del Profesorado de Educación Física. Relación con los Instrumentos de Evaluación. Estudios Pedagógicos, 44(2), 111-126.

https://doi.org/10.4067/S0718-07052018000200111

Córdoba, J. M. et al. (2016). Educación Física Cooperativa, formación permanente y desarrollo profesional. De la escritura colectiva a un relato de vida compartido. Retos. Nuevas tendencias en Educación Física, Deportes y Recreación 29, 264-269.

Fernández, C. (2018). Aprendiendo a desarrollar Sistemas de Evaluación Formativa y Compartida en las prácticas como Maestra de Educación Física en Primaria.Trabajo Fin de Grado. Universidad de Valladolid: Facultad de Educación de Segovia.

Gallardo, F. y Carter, B. (2016). La evaluación formativa y compartida durante el prácticum en la formación inicial del profesorado: Análisis de un caso en Chile. Retos. Nuevas tendencias en Educación Física, Deportes y Recreación, 29, 258-263.

Gallardo-Fuentes, F., López-Pastor, V. M. y Carter-Tuhillier, V. (2018). Efectos de la Aplicación de un Sistema de Evaluación Formativa en la Autopercepción de Competencias Adquiridas en Formación Inicial del Profesorado. Estudios Pedagógicos, 44(2), 55-77. https://doi.org/10.4067/s0718-07052018000200055

Hamodi, C., López, A. T., y López-Pastor, V. M. (2014). La Red de evaluación formativa y compartida en docencia universitaria: Creación, consolidación y líneas de trabajo. REVALUE. Revista de Evaluación Educativa, 3(1).

Hamodi, C., López-Pastor, V. M., y López-Pastor, A. T. (2015). Medios, técnicas e instrumentos de evaluación formativa y compartida del aprendizaje en educación superior. Perfiles Educativos, XXXVII(147), 146-161. https://doi.org/161. 10.1016/j.pe.2015.10.004

Hamodi, C., López-Pastor, V. M., y López, A. (2017). If I experience formative assessment whilst at University will I put it into practice later as a teacher? Formative and shared assessment in Initial Teacher Education (ITE). European Journal of Teacher Education, 4O(2), 171-190. https://doi.org/10.1080/02619768.2017.1281909

Kemmis, S., y McTaggart, R. (1988). Cómo planificar la investigación-acción. Barcelona: Laertes.

Knight, P. (2005). El profesorado de Educación Superior. Formación para la excelencia. Madrid: Narcea.

Latorre, A. (2003). La investigación-acción. Conocer y cambiar la práctica educativa. Barcelona: Graó.

López-Pastor, V. M. (2008). Desarrollando sistemas de evaluación formativa y compartida en la docencia universitaria. Análisis de resultados de su puesta en práctica en la formación inicial del profesorado. European Journal of Teacher Education, 31(3), 293-311.

https://doi. org/10.1080/02619760802208452

López-Pastor, V. M. (Coord.). (2009). La Evaluación Formativa y Compartida en Educación Superior: propuestas, técnicas, instrumentos y experiencias. Madrid: Narcea.

López-Pastor, V. M. (2012). Evaluación Formativa y Compartida en la Universidad: clarificación de conceptos y propuestas de intervención desde la Red Interuniversitaria de Evaluación Formativa. Psychology, Society E' Educatión, 4(1), 117-130. 
López-Pastor, V. M. y Pérez-Pueyo, A. (2017). Buenas prácticas docentes. Evaluación formativa y compartida en educación: Experiencias de éxito en todas las etapas educativas. León: Universidad de León.

López-Pastor, V. M., Hamodi, C. y López A. T. (2016). Evaluación formativa y compartida en educación superior: Buenas prácticas y experiencias desarrolladas por la Red de Evaluación Formativa. En A. Pavié y M. Casas (Eds.), Buenas prácticas de evaluación de aprendizajes en educación superior (pp. 13-43). Santiago de Chile: Ministerio de Educación.

López-Pastor, V. M., Monjas, R. y Manrique, J. C. (2011). Fifteen years of action research as professional development: seeking more collaborative, useful and democratic systems for teachers. Educational Action Research, 19(2), 153-170.

López-Pastor, V. M., Castejón, J., Sicilia-Camacho, A., Navarro, V. y Webb, G. (2011). The process of creating a cross-university network for formative and shared assessment in higher education in Spain and its potential applications. Innovations in Education and Teaching International, 48(1), 79-90.

López-Pastor, V. M., Pérez-Pueyo, A., Barba, J. y Lorente, E. (2016). Percepción del alumnado sobre la utilización de una escala graduada para la autoevaluación y coevaluación de trabajos escritos en la formación inicial del profesorado de educación física (FIPEF). Cultura, Ciencia y Deporte, $11(31)$, 37-50.

López-Pastor, V. M., Ruano, C., Hernangómez, A. et al. (2016). Veinte años de formación permanente del profesorado, investigación-acción y programación por dominios de acción motriz. Retos, Nuevas tendencias en Educación Física, Deporte y Recreación, 29, 270-279.

Manrique-Arribas, J., Vallés, C., y Gea, J. (2012). Resultados generales de la puesta en práctica de 29 casos sobre el desarrollo de sistemas de evaluación formativa en docencia universitaria. PSYE Psychology, Society, E Education, 4(1), 87-102.

Manrique-Arribas, J., López-Pastor, V., Monjas, R. y Real-Rubio, F. (2010). El potencial de los proyectos de aprendizaje tutorado y los sistemas de evaluación formativa en la mejora de la autonomía del alumnado. Una experiencia interdisciplinar en formación inicial del profesorado. Revista Española de Educación Física y Deportes, 14, 39-57.

Martínez-Mínguez, L. (2016). Proyectos de Aprendizaje Tutorados y autoevaluación de competencias profesionales en la formación inicial del profesorado. Retos. Nuevas tendencias en Educación Física, Deportes y Recreación 29, 242-250.

Pérez Serrano, G. (1994). Investigación cualitativa. Retos e interrogantes. I. Métodos. Madrid: La Muralla.

Romero-Martín, M. R., Asún, S. y Chivite, M. T. (2016). La autoevaluación en expresión corporal en formación inicial del profesorado de educación física: un ejemplo de buena práctica. Retos. Nuevas tendencias en Educación Física, Deportes y Recreación, 29, 236-241.

Romero-Martín, R., Castejón-Oliva, F. J., y López-Pastor, V. (2015). Divergencias del alumnado y del profesorado universitario sobre las dificultades para aplicar la evaluación formativa. RELIEVE, 21(1), art. 5. https://doi.org/10.7203/relieve.21.1.5169

Romero-Martín, M., Castejón-Oliva, F., López-Pastor, V. M. y Fraile-Aranda, A. (2017). Evaluación formativa, competencias comunicativas y TIC en la formación del profesorado. Comunicar: Revista Científica de Comunicación y Educación, 52, 73-82. https://doi.org/10.3916/C52-2017-07

Stake, R. (2010). Qualitative research: Studying how things work. Nueva York: Guilford Press.

Simons, H. (2011). El estudio de caso: teoría y práctica. Madrid: Morata. 
Zabalza, M. (2001). La enseñanza universitaria. El escenario y sus protagonistas. Madrid: Narcea.

\section{Cv de los autores}

\section{Cristina Pascual-Arias}

Doctoranda en el Doctorado de Investigación Transdisciplinar en Educación por la Universidad de Valladolid. Docente contratado predoctoral en la Facultad de Educación de Segovia (Universidad de Valladolid). Máster en Investigación en Ciencias Sociales, Educación, Comunicación Audiovisual, Economía y Empresa por la Universidad de Valladolid. Graduada en Educación Infantil por la Universidad de Valladolid. Es miembro de la "Red de Evaluación Formativa y Compartida en Educación". Coordinadora del Proyecto de Innovación Docente de la Universidad de Valladolid: "Evaluación Formativa y Compartida, Transferencia de conocimiento entre Universidad y Escuela”. ORCID-ID: 0000-0002-2781-5600. Email: cristina.pascual@uva.es

\section{Víctor Manuel López-Pastor}

Profesor Titular de Universidad de la Facultad de Educación de Segovia (Universidad de Valladolid). Ha publicado 20 libros y numerosos artículos científicos y profesionales. Sus principales líneas de investigación son: Evaluación Formativa en educación superior, Evaluación Formativa en educación física, Formación del Profesorado, e InvestigaciónAcción. ORCID ID: 0000-0003-2681-9543. Email: vlopez@mpc.uva.es

\section{Carolina Hamodi Galán}

Doctora en Investigación e Innovación en Educación por la Universidad de Valladolid, Máster en Investigación en Ciencias Sociales, Educación, Comunicación Audiovisual, Economía y Empresa por la Universidad Valladolid. Licenciada en Sociología por la Universidad Salamanca y Diplomada en Trabajo Social por la Universidad de Zaragoza. Profesora en el Departamento de Sociología y Trabajo Social de la Universidad de Valladolid (en el Campus de Soria). Ha realizado estancias internacionales en diversos centros de reconocido prestigio, como la Universidad de Cartagena (Cartagena de Indias, Colombia), la Universidad Austral de Chile (Valdivia, Chile), la Universidad Arturo Prat (Iquique, Chile), la Universidad de Buenos Aires (CABA, Argentina), la Universidad de an Carlos (Puerto Barrios, Guatemala) o la Università degli Studi di Firenze (Florencia, Italia). Es miembro de diferentes grupos de investigación, tanto nacionales como internacionales: la "Red de Evaluación Formativa y Compartida en Docencia Universitaria", el grupo de investigación "Educación, Universidad y Sociedad" de la Universidad de Cartagena (Colombia) reconocido por COLCIENCIAS (departamento Administrativo de Ciencia, Tecnología e Innovación del gobierno de Colombia) y el Grupo de Investigación “Ciencias Sociales Aplicadas” de la Universidad de Valladolid (España). ORCID ID: 0000-0002-0965-8410. Email: carolina.hamodi@uva.es 